Supporting Information

\title{
Cytosolic Delivery of Functional Proteins In Vitro through Tunable Gigahertz Acoustics
}

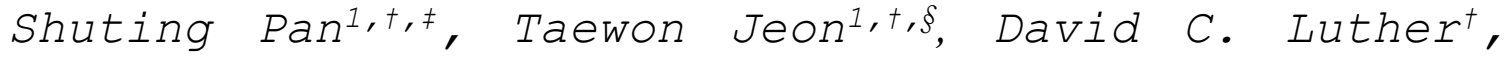 \\ Xuexin Duan*,, Vincent M. Rotello*, + \\ $\dagger$ Department of Chemistry, University of \\ Massachusetts, 710 North Pleasant Street, Amherst, \\ Massachusetts 01003, United States \\ * State Key Laboratory of Precision Measuring \\ Technology \& Instruments, college of Precision \\ Instrument and Optoelectronics Engineering, Tianjin \\ University, Tianjin 300072, China.
}

$\S$ Molecular and Cellular Biology Graduate Program, University of Massachusetts Amherst, 710 N. Pleasant St., Amherst, United States

\footnotetext{
* E-mail: rotellodchem.umass.edu; xduandtju.edu.cn.

Tel./Fax: +862227401002 (X.D.) .

1 These authors contributed equally.
} 
Supplementary I - GHz acoustic streaming setup

After fabrication, the electrical property of $\mathrm{GHz}$ acoustic resonator was tested by a vector network analyzer (Agilent N50171C), and then sealed on a $\mathrm{T}-$ shape circuit board, which is well compatible with 96-well plate. The setup and layout are schematically shown in Figure s1. Herein, RF signal was generated by a signal generator (Agilent, N5181A), amplified by a power amplifier (Mini-Circuits, ZHL-5 W-422), and applied to the resonator. The isolator is utilized to prevent RF signal feedback.

(a)

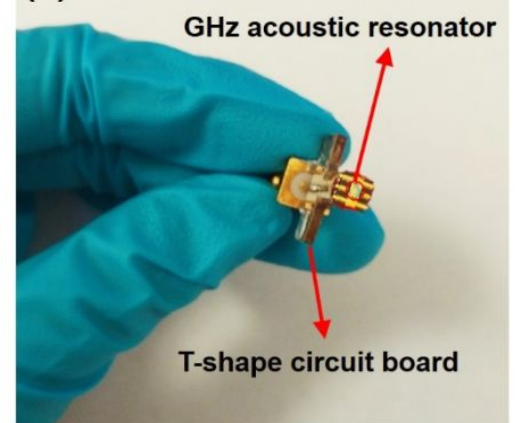

(b) Signal Generator

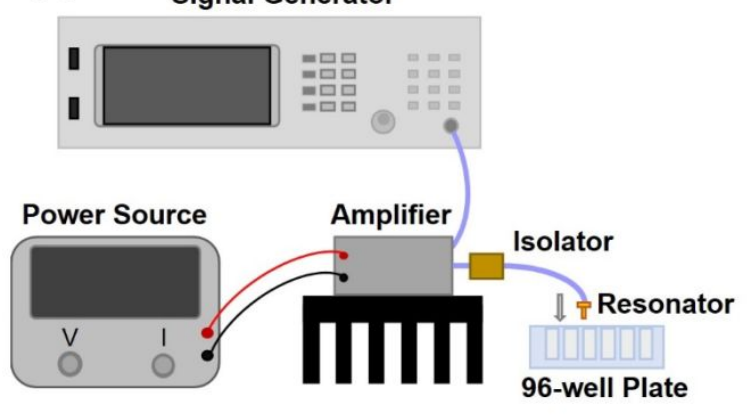

Figure S1. (a) Photography of a GHz acoustic resonator sealed on a T-shape circuit board. scheme of experimental setup.

Supplementary II - GHz acoustic streaming and the induced cell membrane deformation

Due to the nonlinear fluid dynamics, the periodic acoustic wave suffers from acoustic attenuation when coupled with liquid, which results in acoustic streaming. In other words, the acoustic energy is radiated into the liquid and induces directional flow in the fluid. When the jet flow reaches the substrate interface, it is forced to flush horizontally. This flushing acoustic streaming finally applies lateral force on the cells. Figure s2 shows the cell deformation observed by high speed confocal 
microscopy Z-stacking (Figure s2a). The cells were cultured at the bottom of a typical 96 well plate, where the acoustic device is mounted on top. The cell membrane was stained with wheat germ agglutinin (Alexa Fluor 647 conjugate) for $20 \mathrm{~min}$. As shown in Figure $\mathbf{S} \mathbf{2 b}$ and $\mathbf{c}$, when GHz acoustic streaming applied, the profile of cell was changed and the cell membrane was extended. Such change is schematically depicted by the change of cell profile (Figure s2d) .

a

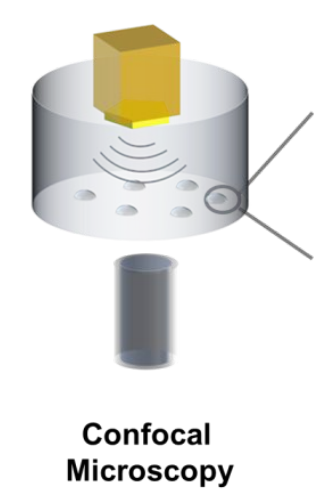

b c
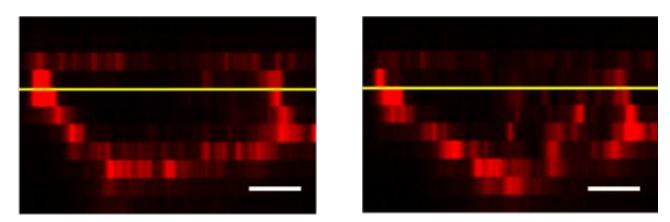

d

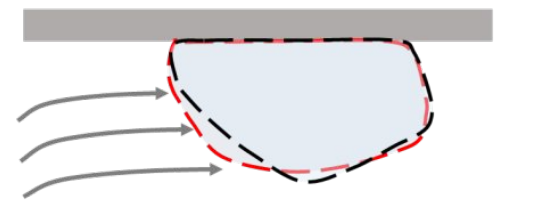

Acoustic Streaming

Figure S2. Cell membrane deformation induced by the $\mathrm{GHz}$ acoustic streaming. (a) set up for photographing the cell membrane deformation. The profile of cell before (b) and after (c) GHz acoustic streaming. Scale bar: 5 um. (d) Scheme of the working mechanism of GHz acoustics system.

Supplementary III - Localization of delivered proteins in nucleus

Confocal microscopy Z-stacking was utilized to show the cytosolic localization of delivered +25GFP, which can be found in supplementary video 1 .

Supplementary IV - Confocal images for optimal working conditions

Figure $\mathbf{s} 3$ shows confocal images for optimal working conditions; the mean fluorescence intensity 
of these images is plotted in Figure 3 in main text. From the confocal images, we can conclude that the delivery area and efficiency is impoved according with the increasing applied acoustic power from 200 $\mathrm{mW}$ to $400 \mathrm{~mW}$, while there is no further improvement with increased power above $400 \mathrm{~mW}$.

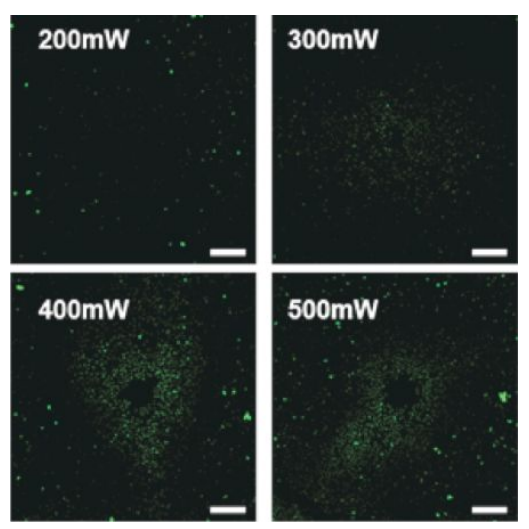

Figure S3. Fluorescent confocal images of HeLa cells after acoustic treatment at varied power $(200 \mathrm{~mW}$, $300 \mathrm{~mW}, 400 \mathrm{~mW}$ and $500 \mathrm{~mW}$ ) for $20 \mathrm{~min}$.

Supplementary $\mathbf{V}-$ Investigation of cytosolic delivery of proteins with different surface charge

This part aims to further confirm the mechanism of this GHz acoustics-assisted method. Due to that the mechanism is through gentle deformation of cell membrane instead of large-scale pores on the membrane, the negatively-charged proteins could not be delivered into cells. In this case, as shown in Figure s4, +25GFP was successfully delivered into cells using GHz acoustics, while wild type GFP with acoustic treatment showed no delivery. 

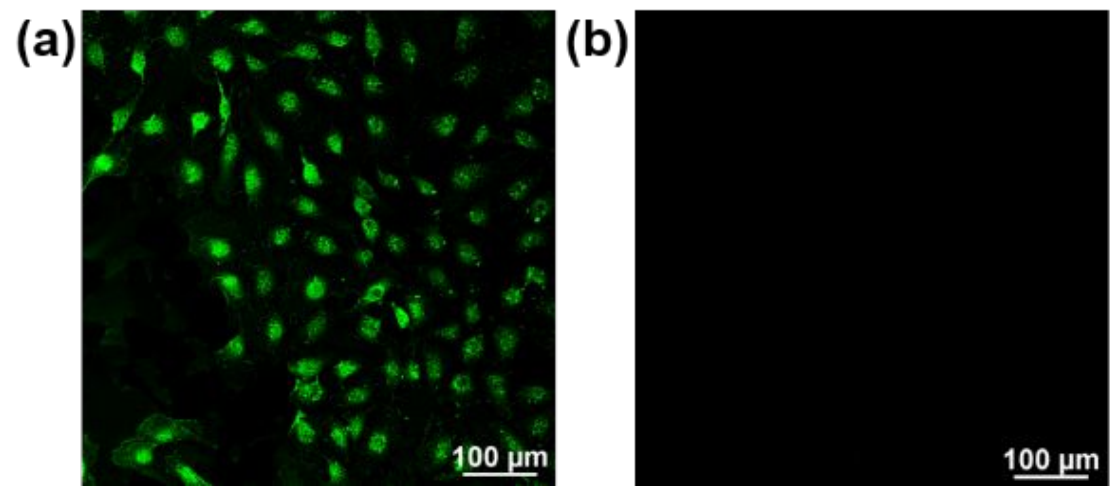

Figure s4. Confocal images showing cellular of (a) +25GFP and (b) wild type GFP. 